\title{
THE RADIOASTRON PROJECT
}

\author{
N. S. Kardashev and V. I. Slysh \\ Space Research Institute, U.S.S.R. Academy of Sciences \\ Moscow, U.S.S.R.
}

\begin{abstract}
The RADIOASTRON mission (Andreyanov et al. 1986) is designed to achieve angular resolutions as fine as 6 microarcsec and will be used to study radio sources with very high brightness temperature. It will form an orbiting radio interferometer between a satellite radio telescope $10 \mathrm{~m}$ in diameter and several large ground-based radio telescopes. The orbit of the satellite makes it possible to have baselines from several thousands to $80,000 \mathrm{~km}$. The satellite will be equipped with four dual polarization receivers at $327,1665,4830$, and $22235 \mathrm{MHz}$, with local oscillators phase-locked to a ground-based hydrogen maser frequency standard via an S-band microwave link. The IF signals from the receivers will be transmitted to the ground by an $\mathrm{X}$-band high data rate link and recorded on magnetic tape in the VLBA format. Participation of large radio telescopes from many countries is envisaged and encouraged.
\end{abstract}

\section{INTRODUCTION}

The angular resolution of ground-based VLBI systems such as the European VLBI Network (EVN) or the Very Long Baseline Array (VLBA), which is under construction, is limited by the dimensions of the Earth; the baseline of any ground-based interferometer cannot exceed $12,000 \mathrm{~km}$, which limits the angular resolution to about 0.1 milliarcsecond at $6 \mathrm{~cm}$. There are two ways of increasing the angular resolution further: one is to go to millimeter and submillimeter wavelengths; another is to go to space to increase the baseline length. The former way encounters certain problems caused by atmospheric phase fluctuations; the latter way is more straightforward and presents no limitations in principle to the baseline length. Microarcsecond angular resolution will allow us to study the following phenomena:

- flare activity of nearby stars;

- planets around nearby radio emitting stars;

- measurement of distance to pulsars and other compact galactic sources through their parallax and proper motion;

- light cylinder zone of pulsars and the ejection of relativistic particles;

- structure of binary systems, neutron stars, or black hole-stars and the ejection of relativistic particles from them;

- structure and dynamics of maser radio sources in star-forming regions; tur- 
bulent structure of interstellar matter and scintillation of radio sources;

- candidate large black hole at the galactic center;

- acceleration and collimated ejection of relativistic particles by supermassive AGN's and QSO's;

- super-Compton emission and superluminal motion;

- cosmological evolution of compact extragalactic radio sources;

- determination of fundamental cosmological parameters and of the nature of hidden mass.

Since the flux density sensitivity of a space interferometer and a ground-based interferometer is approximately the same, the space interferometer will be sensitive to much higher brightness temperatures of radio sources, because this sensitivity scales as baseline length squared. Although brightness temperatures of synchrotron emission sources must be limited to $10^{12} \mathrm{~K}$ (Kellermann and Pauliny-Toth 1969, 1981), there is evidence of Doppler boosting, which increases the brightness temperature of AGN's and QSO's by a factor of $\gamma^{3+\alpha}$, where $\gamma$ is the Doppler factor, and $\alpha$ is the spectral index. For $\gamma \sim 10^{3}, \alpha \sim 0$, the brightness temperature may be as high as $10^{21} \mathrm{~K}$. Nonsynchrotron radio sources such as $\mathrm{OH}$ and $\mathrm{H}_{2} \mathrm{O}$ masers and pulsars may also have very high brightness temperatures. Some $\mathrm{OH}$ masers have been measured to have brightness temperatures as high as $4 \times 10^{12} \mathrm{~K}$, while $\mathrm{H}_{2} \mathrm{O}$ masers reach $10^{16} \mathrm{~K}$. The brightness temperature of pulsars is estimated to reach $10^{30} \mathrm{~K}$.

Another advantage of the space radio interferometer over a ground-based array is its ability to measure much smaller angular motions related to superluminal motion in quasars. As shown in Figure 1, angular resolution from space at $1.35 \mathrm{~cm}$ might be high enough to discriminate between different cosmological models through measurement of proper motion of large samples of superluminal radio sources with large redshifts (Cohen et al. 1987).

\section{ORBITAL PARAMETERS AND U-V PLANE COVERAGE}

The RADIOASTRON project is aimed at the creation of a space-Earth radio interferometer that will be able to realize angular resolution as fine as 6 microarcseconds. It will consist of one radio telescope on a satellite circling around the Earth on a highly eccentric orbit and several large ground-based radio telescopes operating in the standard VLBI mode. The orbital parameters are given in Table 1.

Table 1. Orbital parameters of the RADIOASTON satellite

$\begin{array}{ll}\text { Period } & P=24^{h} \\ \text { Semimajor axis } & a=42,000 \mathrm{~km} \\ \text { Semiminor axis } & b=26,000 \mathrm{~km} \\ \text { Eccentricity } & \varepsilon=0.8 \\ \text { Inclination } & i=67^{\circ} \\ \text { Perigee radius } & r_{p}=9,000 \mathrm{~km} \\ \text { Apogee radius } & r_{a}=75,000 \mathrm{~km}\end{array}$




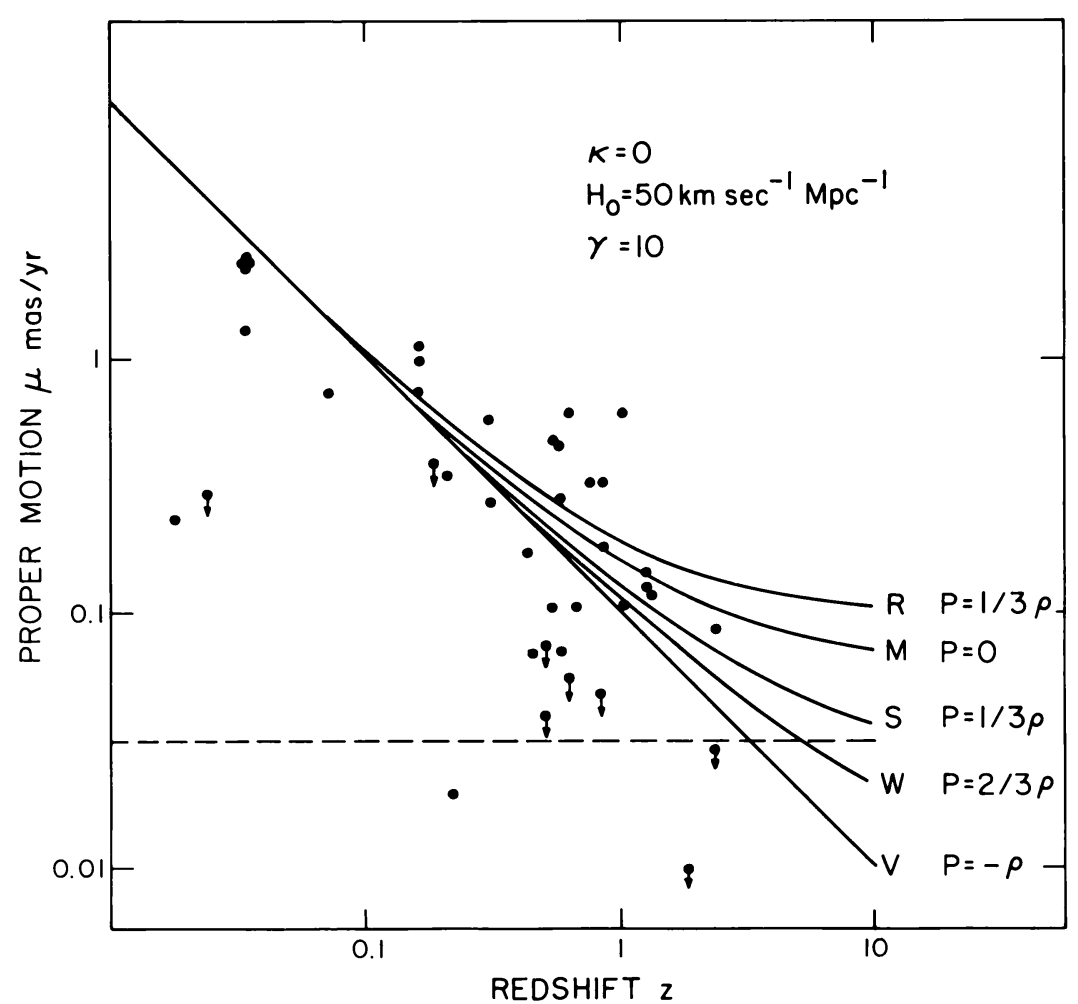

Figure 1. Proper motion of superluminal components versus redshift. The data points are from Cohen et al. (1987). Solid lines show the proper motion-redshift relation for different cosmological models: $R$-cosmological model with dominating relativistic matter (equation of state indicated on the right side); $M$-the same for nonrelativistic matter (pressure equals zero); $S$-the same for infinite strings; $W$-the same for infinite walls; $V$ - the same for vacuum. The curvature, the Hubble constant, and Lorentz-factor used for plotting the solid lines are 0 , $50 \mathrm{~km} \mathrm{~s}^{-1} \mathrm{Mpc}^{-1}$, and 10, respectively. The dashed line gives the sensitivity of RADIOASTRON at $1.35 \mathrm{~cm}$ wavelength.

The orbit is schematically shown in Figure 2. uv-plane coverage of such an interferometer may be rather poor for certain projections, as shown in Figure 3, and is not as well suited for construction of images as the VLBA system or the QUASAT project. New data processing algorithms must be designed to deal with this poor uv-coverage.

\section{BASIC COMPONENTS OF THE RADIOASTRON SYSTEM}

An overall schematic diagram of the RADIOASTRON system is shown in Figure 4. We describe briefly some of the major components. 
436

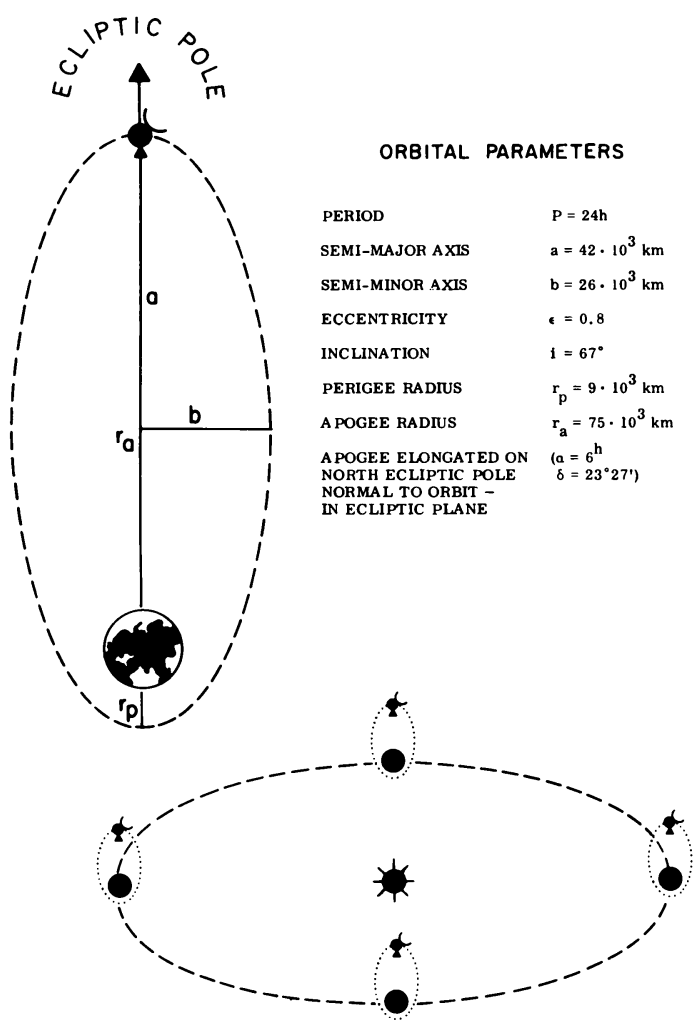

Figure 2. Proposed orbit of the RADIOASTRON satellite.

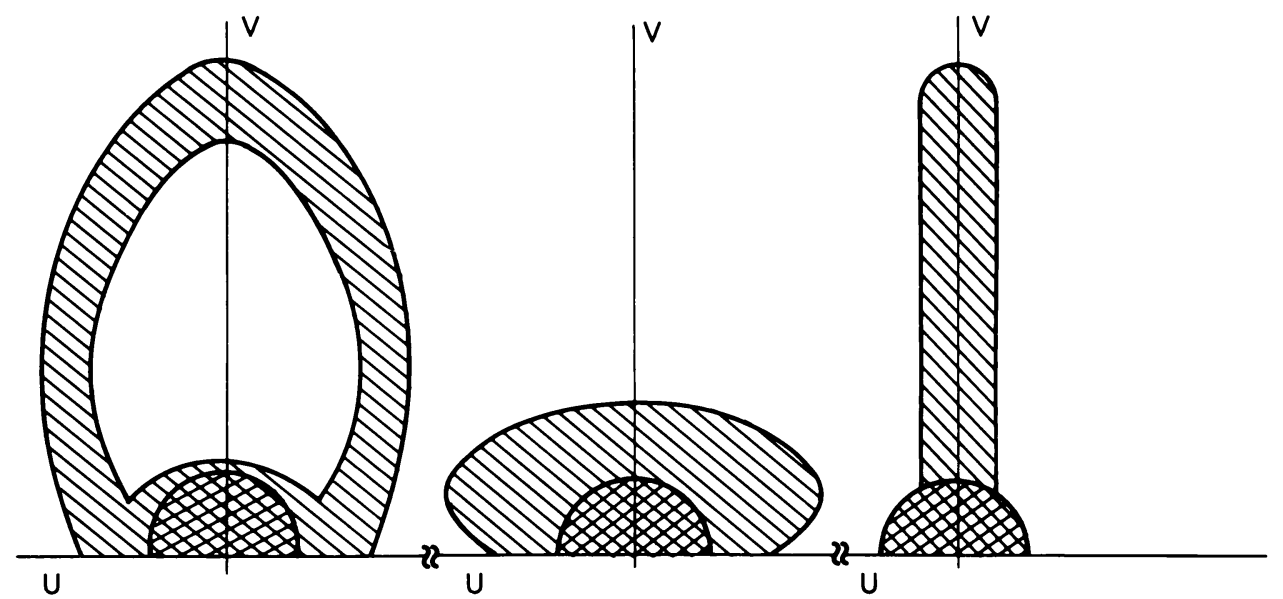

Figure 3. uv-plane coverage for a VLBI network including RADIOASTRON. Left: for a source lying in the direction normal to the orbital plane. Center: the same for a direction to the source making an angle of $75^{\circ}$ with the major axis. Right: the same for a direction to the source coinciding with the minor axis. The double crosshatched area near the origin corresponds to uv-plane coverage of the ground-based VLBI network without RADIOASTRON. 


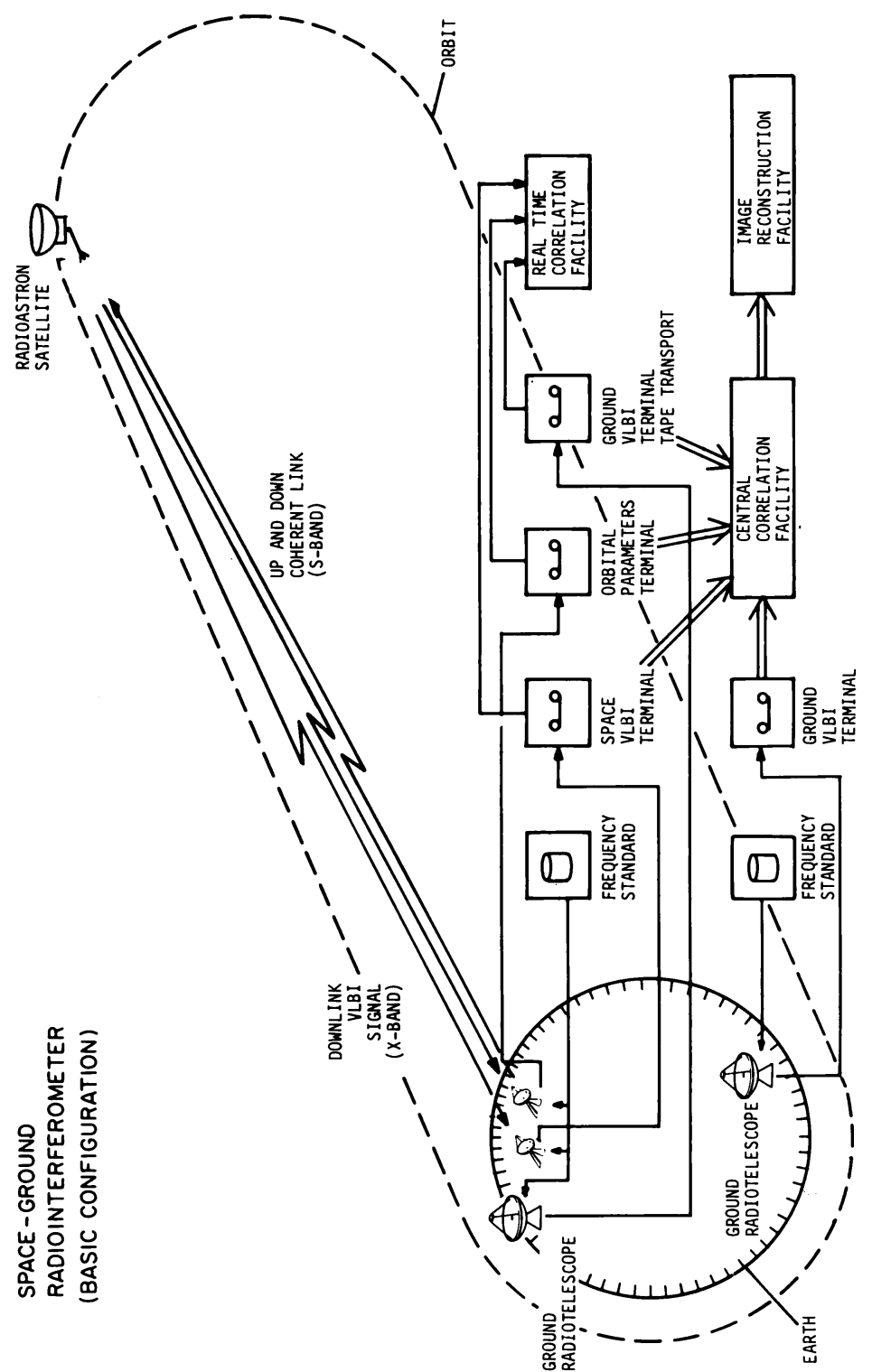

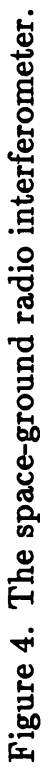




\subsection{Antenna}

The satellite radio telescope, shown in Figure 5, will be a prime focus system with a deployable parabolic reflector $10 \mathrm{~m}$ in diameter. The reflector has a fixed inner part of 3-m diameter and 24 unfoldable panels. The focal package is attached to the inner 3-m part of the reflector with four focal support legs. Feeds and receiver front-ends are located in the focal package. The reflector is made of reinforced carbon fiber and has an rms surface accuracy of $0.5 \mathrm{~mm}$.

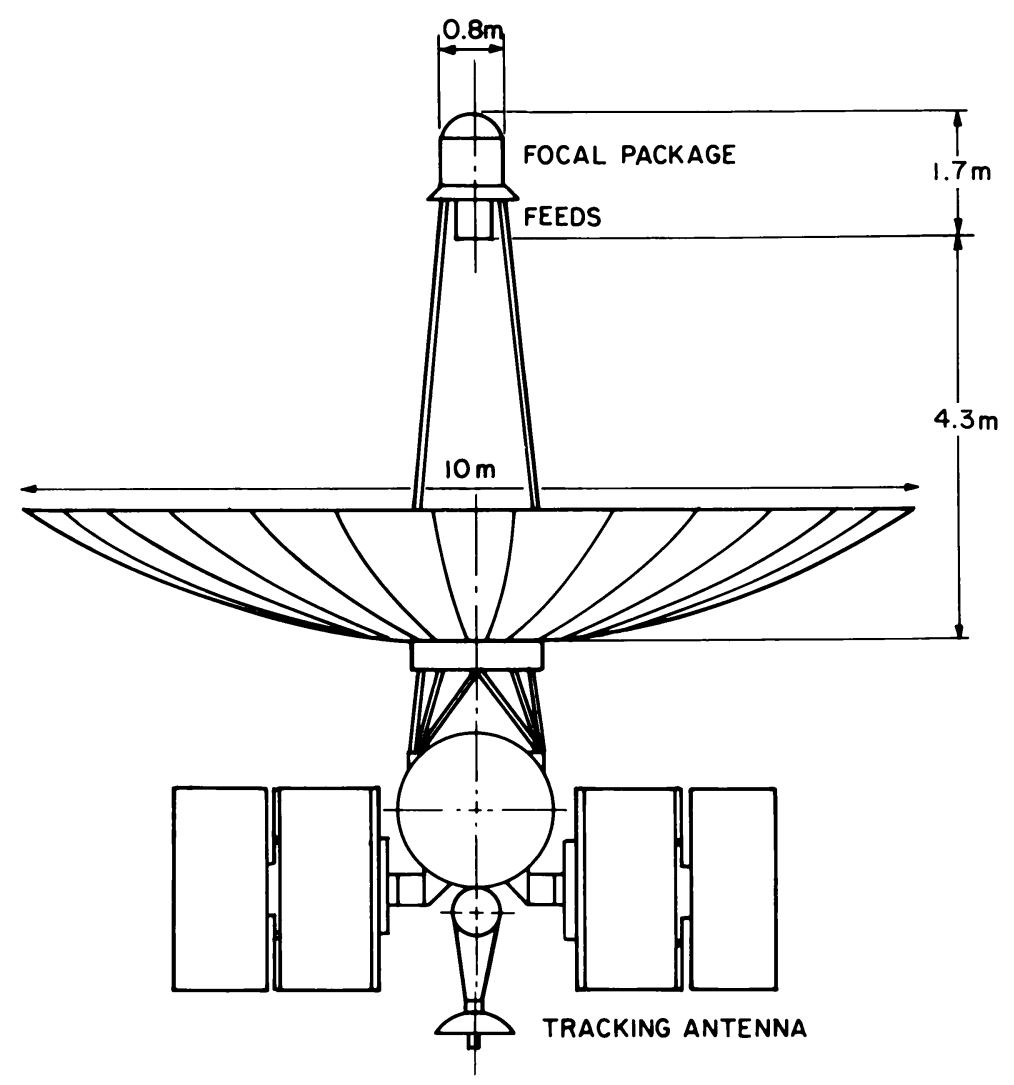

Figure 5. The RADIOASTRON satellite.

\subsection{Receivers}

Four dual circular polarization receivers will be provided on the satellite. Table 2 shows the basic parameters of the receivers and the expected sensitivity and angular resolution of the RADIOASTRON satellite in conjunction with a 70-m diameter ground-based radio telescope.

The receivers will have cooled low noise transistor amplifiers as front-ends (except at $327 \mathrm{MHz}$, where the receiver will be uncooled). The refrigeration system 
Table 2. Parameters of the RADIOASTRON system

\begin{tabular}{lcccr}
\hline $\begin{array}{c}\text { Frequency } \\
(\mathrm{MHz})\end{array}$ & $\begin{array}{c}\text { Bandwidth } \\
(\mathrm{MHz})\end{array}$ & $\begin{array}{c}\text { System Noise } \\
\text { Temperature } \\
(\mathrm{K})\end{array}$ & $\begin{array}{c}\text { Flux Density } \\
\text { Sensitivity } \\
(\mathrm{mJy})\end{array}$ & $\begin{array}{c}\text { Angular } \\
\text { Resolution } \\
(\mu \mathrm{ma})^{2}\end{array}$ \\
\hline 327 & 4 & 80 & 10 & 400 \\
1665 & 32 & 50 & 3.5 & 80 \\
4830 & 32 & 50 & 3.5 & 24 \\
22235 & 32 & 70 & 4.0 & 6 \\
\hline
\end{tabular}

$15 \times$ rms, averaged ove: two circular polarizations, 18 hours integration time.

$20.2 \lambda / B_{\max }, B_{\max }=80,000 \mathrm{~km}$. This criterion is used throughout the paper. It assumes a moderate signal-to-noise ratio such that details smaller than the nominal diffraction-limited beam size of $\sim 0.5 \lambda / D$ can be discerned.

relies on radiation cooling to $150 \mathrm{~K}$ and a closed-cycle cooling machine, which will cool the front-ends to $80 \mathrm{~K}$. Each receiver will have its own feed in the focal plane of the antenna with polarization splitters and calibration system. The coma losses due to the offset positions of the three feeds (22 GHz feed will be placed at the focus) will not exceed $0.2 \mathrm{db}$. The cooled front-ends and polarization splitters will be located in a separate focal plane assembly connected by RF cables and waveguides to the focal package where the receivers are located.

\subsection{Local oscillator}

All local oscillator frequencies will be derived from a coherent frequency synthesizer, which will be phase-locked to a ground-based hydrogen maser frequency standard by a two-way (up and down) S-band link that utilizes an omnidirectional antenna on the satellite. As a backup, an on-board rubidium frequency standard with a stability of $10^{-12}$ will be available.

\subsection{IF and video}

The receivers have standard $500 \mathrm{MHz}$ IF amplifiers and IF-to-video converters. Two independent channels for both circular polarizations are converted to the baseband and then sampled. The sampling rate is $64 \mathrm{mbits} / \mathrm{sec}$ in each channel, which allows for two-level sampling of two $32 \mathrm{MHz}$ bandwidth channels or four-level sampling of two $16 \mathrm{MHz}$ bandwidth channels.

\subsection{Down-link}

The down-link to the ground station is at X-band $(8.2 \mathrm{GHz})$ with a two-channel transmitter and high-gain parabolic antenna. The antenna for the down-link has a diameter of $1 \mathrm{~m}$ and can be pointed to the ground within 0-120 from the antisolar direction in one plane and $\pm 30^{\circ}$ for the perpendicular plane, with a pointing error less than $0.5^{\circ}$. 


\subsection{Ground-based station}

The ground-based station of the RADIOASTRON mission consists of one transmitting (command and S-band local oscillator up-link) antenna and two receiving antennas (S-band local oscillator down-link and X-band high-data rate down-link). The 70-m antenna will be used as a radio telescope to receive signals from a radio source. Both $\mathrm{X}$-band data and $70-\mathrm{m}$ radio telescope outputs will be recorded on two tape recorders in the VLBA format. Simultaneous observations by telescopes outside the Soviet Union will be recorded in a VLBA-compatible format; participation of large radio telescopes from Australia, Canada, Europe, India, Japan, and the U.S. is expected. Tapes of the data from the satellite and all participating radio telescopes will be processed on the NRAO VLBA correlation facilities, on the European VLBI Network correlator, and on the facilities of the Space Research Institute in Moscow.

\section{SUMMARY}

The RADIOASTRON mission is scheduled to fly in 1991 and will have a lifetime of two years. Realization of this project will be possible with broad international cooperation, both through a combined effort to construct the satellite radio telescope and through participation of as many large radio telescopes as possible. It will be possible to achieve sufficient sensitivity even with the modest $10-\mathrm{m}$ diameter satellite radio telescope to undertake many important investigations. An increase of angular resolution by an order of magnitude compared to ground-based VLBI is only a first step for future improvements of many orders of magnitude using space interferometers. However, this first step will permit the solution of many of the astronomical problems mentioned earlier.

\section{REFERENCES}

Andreyanov, W., et al. 1986, Astron. Zh., 63, 850.

Cohen, M.H., Barthel, P.D., Pearson, T.J., and Zensus, J.A. 1987, The Impact of VLBI on Astrophysics and Geophysics, Proc. of IAU Symposium 129 (this volume).

Kellermann, K.I., and Pauliny-Toth, I.I.K. 1969, Ap. J. (Letters), 155, L71.

Kellermann, K.I., and Pauliny-Toth, I.I.K. 1981, Ann. Rev. Ast. Astrophy., 19, 373. 